\title{
Wind Turbine Blade Monitoring with Brillouin-Based Fiber-Optic Sensors
}

\author{
Agnese Coscetta, ${ }^{1}$ Aldo Minardo, ${ }^{1}$ Lucio Olivares, ${ }^{2}$ Maurizio Mirabile, ${ }^{3}$ Mario Longo, \\ Michele Damiano, ${ }^{4}$ and Luigi Zeni ${ }^{1}$ \\ ${ }^{1}$ Department of Industrial and Information Engineering, Seconda Università di Napoli, Via Roma 29, 81031 Aversa, Italy \\ ${ }^{2}$ Dipartimento di Ingegneria Civile Design Edilizia e Ambiente, Seconda Università di Napoli, Via Roma 29, 81031 Aversa, Italy \\ ${ }^{3}$ Optosensing SRL, Via Adriano Olivetti 1, 80078 Pozzuoli, Italy \\ ${ }^{4}$ Cartflow SRL, Via Centauro 1, 81030 Castel Volturno, Italy
}

Correspondence should be addressed to Agnese Coscetta; agnese.coscetta@unina2.it

Received 17 October 2016; Revised 16 February 2017; Accepted 1 March 2017; Published 13 March 2017

Academic Editor: Hai Xiao

Copyright (C) 2017 Agnese Coscetta et al. This is an open access article distributed under the Creative Commons Attribution License, which permits unrestricted use, distribution, and reproduction in any medium, provided the original work is properly cited.

Wind turbine (WT) blade is one of the most important components in WTs, as it is the key component for receiving wind energy and has direct influence on WT operation stability. As the size of modern turbine blade increases, condition monitoring and maintenance of blades become more important. Strain detection is one of the most effective methods to monitor blade conditions. In this paper, a distributed fiber-optic strain sensor is used for blade monitoring. Preliminary experimental tests have been carried out over a $14 \mathrm{~m}$ long WT composite blade, demonstrating the possibility of performing distributed strain and vibration measurements.

\section{Introduction}

Wind turbines (WTs) are increasingly populating the landscape, offering an additional source of electric power. Increased harnessing of wind power provides renewable energy reducing reliance on fossil fuels. Over the past few years, WTs have become larger, and the forces acting on the blades have increased, leading to a higher possibility of fatigue failures such as broken WT blades or tower strikes. This causes the industry to have a high focus on structural health monitoring (SHM) of WT components to reduce failure and maintenance costs.

Distributed optical fiber sensors offer the ability to measure temperatures and/or strains at thousands of points along a single fiber $[1,2]$. In particular, they constitute a relatively new technology for monitoring the performance of WT blades [3-6]. For this application, the main advantage of distributed optical fiber sensors, especially those based on stimulated Brillouin scattering, consists in the capability of monitoring the strain over the entire surface with high (submeter or even $\mathrm{cm}$ scale) spatial resolution. Therefore, detection technology using Brillouin distributed fiber-optic sensors may be used to find hidden trouble in the blade and estimating the location and size of fatigue damage. Also, one single Brillouin interrogator can be used to collect monitoring data of several turbine blades. Finally, the same monitoring system can be used to estimate the dynamic properties of the blades, such as natural frequencies and damping properties, which are essential parameters for the dynamic behavior and structural integrity of the entire WT [7].

In this paper, the results of two test cases carried out at the Cartflow site in Castel Volturno (Italy) are shown, demonstrating the capability of Brillouin Optical Time-Domain Analysis (BOTDA) sensors to realize distributed static and dynamic strain measurements. The proposed system provides real-time in situ monitoring. In addition, the system may be used to validate WT blade structural models, analyzing the structure not only nondestructively but also without implanting intrusive electrical elements and transducers into the structure. Finally, the proposed technology may be even used to improve the blade design tools and the manufacturing process. 


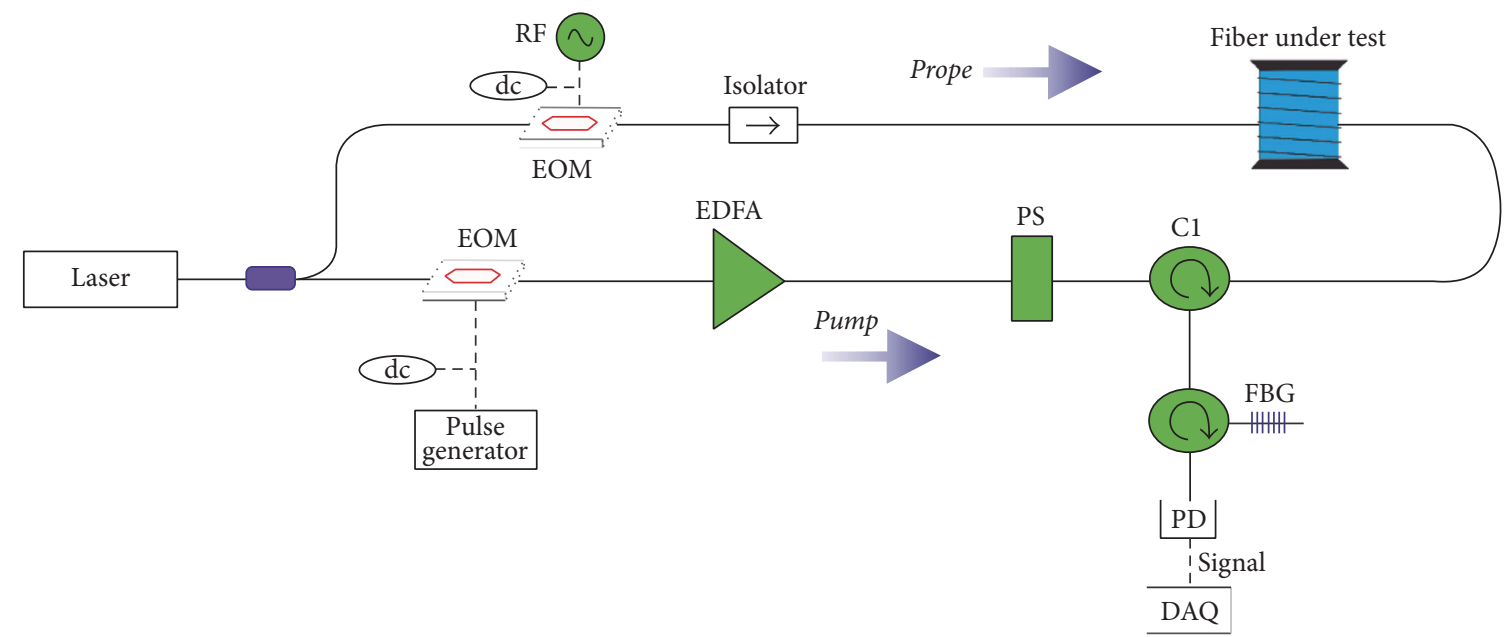

FIGURE 1: Schematic of the BOTDA measurement setup.

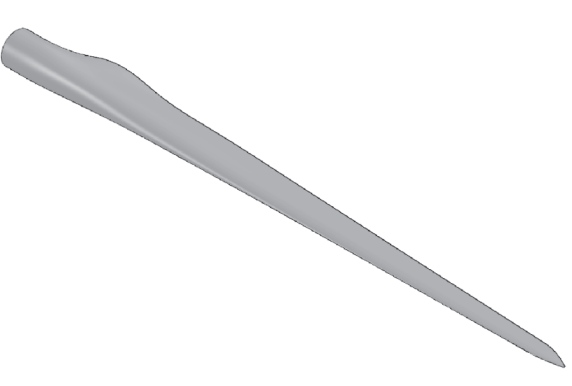

(a)

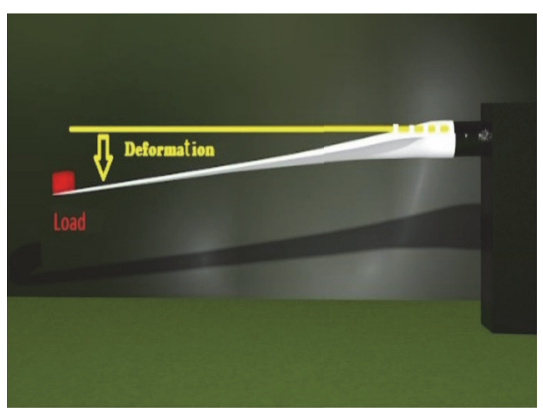

(b)

Figure 2: (a) CAD image of the WT used for distributed static strain measurements; (b) schematics of the load conditions used during experimental tests.

\section{Experimental Results}

The experimental results reported in this paper have been conducted exploiting stimulated Brillouin scattering (SBS) in single-mode optical fibers. In brief, two counterpropagating light waves exchange energy along the fiber, in a measure depending on their frequency offset. If the offset falls within a specific range, the radiation at higher frequency (pump wave) transfers energy to that at lower frequency (Stokes wave). Sensing is based on the fact that the frequency difference at which the Stokes wave experiences the maximum gain, known as Brillouin frequency shift (BFS), and varies depending on the mechanical and thermal state of the fiber. In particular, in conventional silica fibers, the BFS increases with both temperature and strain. Spatial resolution, that is, the ability to measure deformation and temperature changes with spatial continuity, can be achieved by a pulsed pump beam: in this way, the interaction takes place along successive sections of the fiber as the pump pulse propagates down the fiber under test. By recording the intensity of the transmitted Stokes radiation as a function of time, the Brillouin gain can be traced at each section. Brillouin time-domain signals are acquired for a range of pump-probe frequency shifts, in order to retrieve the Brillouin gain spectrum (BGS) at each location. Finally, the peak frequency of each BGS (i.e., the BFS) is retrieved by peak searching and is converted into strain or temperature, through the use of appropriate calibration coefficients.

The BOTDA interrogator used for the strain measurements implements the setup shown in Figure 1. The light from a distributed feedback laser diode (DFB-LD) operating at $1.55 \mu \mathrm{m}$ wavelength is split into two distinct arms to generate the pump and probe beams. The lower branch in the figure shows the generation of the pulsed pump, while the upper branch shows the generation of the probe signal through double-sideband suppressed-carrier modulation. A polarization scrambler (PS) is employed to average out the Brillouin gain fluctuations associated with variations of state of polarization of the interacting beams. Finally, the fiber Bragg grating (FBG) selects the sideband at lower frequency (Stokes component), while the optical beam reflected by the FBG is detected by a photodetector (PD) and acquired by a data acquisition system (DAQ).

A first test was performed, aimed to measure the deformation of a WT composite blade at different load conditions. The blade was $14 \mathrm{~m}$ long and configured as a simple cantilever beam, with the root end fixed to a rotor and the load applied to the opposite end in the flapwise direction. We show in Figure 2(a) a computer-aided design (CAD) image 


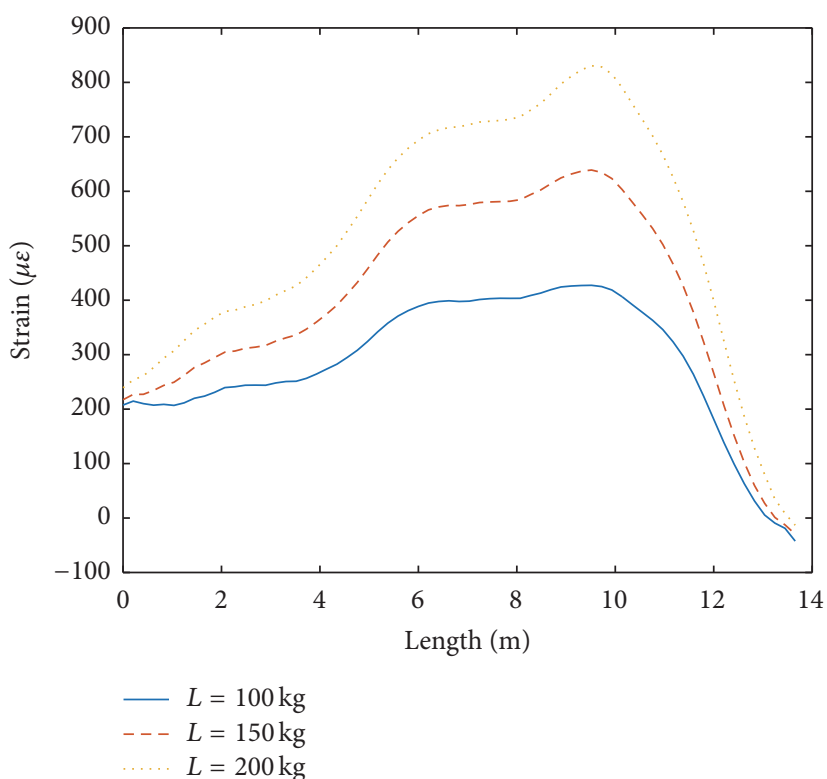

(a)

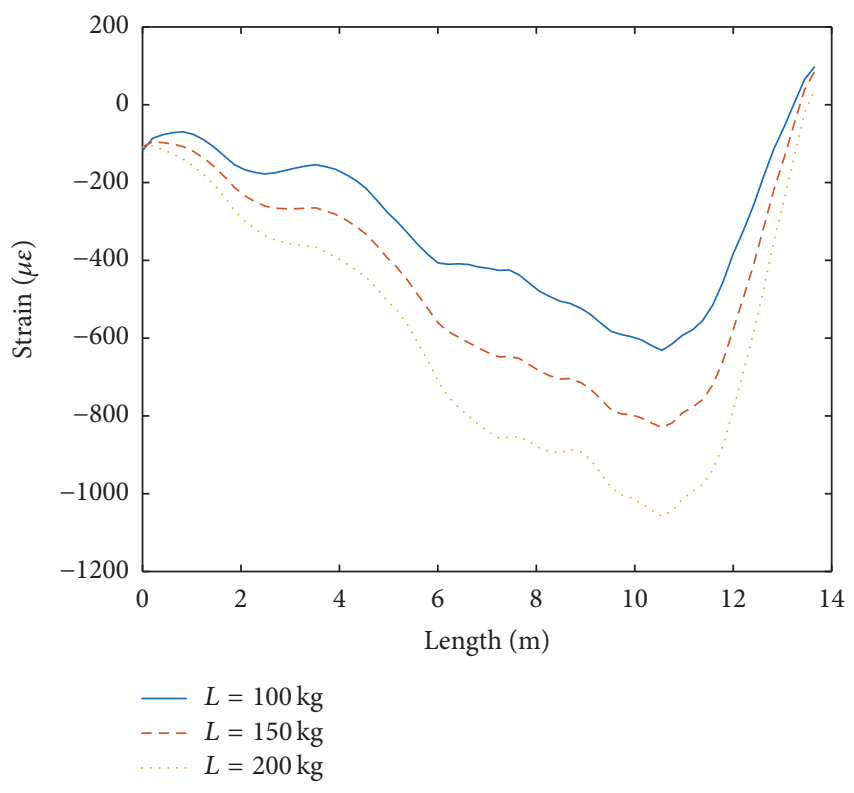

(b)

FIGURE 3: Static strain along the pressure side (a) and suction side (b) path of the blade.

of the WT blade, while Figure 2(b) depicts the experimental arrangement used for static tests. A single-mode optical fiber was attached along the middle line of both blade surfaces, in a loop configuration, by use of a commercial epoxy adhesive. A short loop of loose fiber was left between the bonded fiber segments, in correspondence with the loaded end of the blade.

The fiber was then connected to the interrogator, in order to perform the distributed strain measurements. The acquisitions were performed at a spatial resolution of $1 \mathrm{~m}$, a digital spatial step of $20 \mathrm{~cm}$ and a number of averages equal to 2048 , leading to an overall acquisition time of 70 s. Spatial resolution refers to the minimum size of strain perturbation which can be correctly retrieved by our sensor and is directly related to the duration of the pump optical pulses, while digital spatial step corresponds to the distance between consecutive sensing points and is related to the sampling rate of the data acquisition system.

After acquisition of the reference profile (i.e., the BFS profile in absence of load), a number of distributed strain measurements were performed changing, each time, the applied load. In particular, we changed the load weight by adding $50 \mathrm{~kg}$ cement bags. The results are reported in Figure 3, where a BFS/strain coefficient of $47 \mathrm{kHz} / \mu \varepsilon$ was adopted for transduction. Note that the abscissa $z=0$ corresponds to the fixed end of the blade. We note that the strain sign is correctly captured by the sensor, with the fiber in the pressure side (i.e., glued on the top surface of the blade) being mostly subjected to tensile strain and the fiber in the suction side (i.e., glued on the bottom surface) mostly subjected to compressive strain.

The complex profiles of the strain can be attributed partly to the cantilevered configuration of the blade during the tests and partly to the complex geometry of the blade itself. In particular, the thickness of the blade changes continuously



FIgURE 4: Maximum tensile strain measured by the sensor, as a function of the load.

in both spanwise and chordwise direction. Also blade chord distribution changes, increasing from $825 \mathrm{~mm}$ at the root up to maximum chord of $1180 \mathrm{~mm}$ at $z=2030 \mathrm{~mm}$ and then decreasing gradually along the span, down to $50 \mathrm{~mm}$ at the tip. From Figure 3, it is seen that maximum tensile (and compressive) strain is reached at about $10 \mathrm{~m}$ from the fixed end, as a consequence of the stiffness distribution in the load direction.

Despite the limited number of test loads, the linearity between measured strain and applied load can be appreciated from Figure 3, analyzing any positon along the blade. In particular, we plot in Figure 4 the maximum tensile strain 


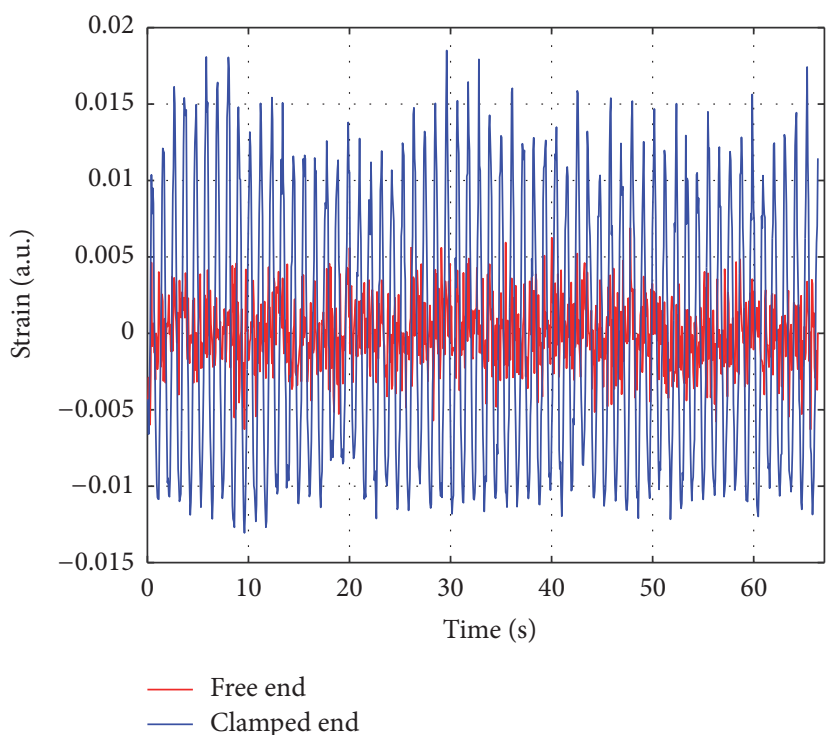

(a)

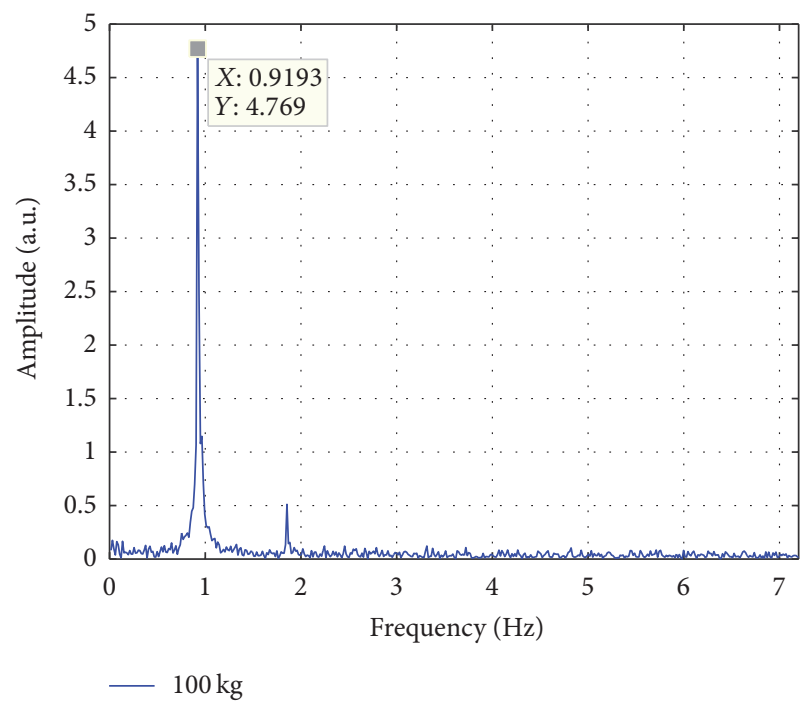

(b)

Figure 5: (a) Dynamic strain measurement along the blade excited at its first resonance, acquired at the clamped end (blue curve) and the free end (red curve). (b) Corresponding Fourier spectra (load weight $=100 \mathrm{~kg}$ ).

recorded during the tests, as a function of the load. As expected, a good linear relationship is observed between these two quantities.

The second test case was aimed to demonstrate the capability of the reading unit to perform dynamic strain measurements. The dynamic strain was acquired using the same equipment, operated in order to implement the socalled slope-assisted BOTDA [8-10]. In brief, the slopeassisted method consists in fixing the pump-probe frequency shift at a value lying in the middle of the left or right slope of the BGS. In this way, any variation of the BFS due to strain is dynamically mapped in changes in the intensity of the received probe wave. As the method avoids the timeconsuming scanning of the pump-probe frequency shift, it provides fast acquisition speeds.

The blade was put in vibration at its first resonance exploiting an electrical motor positioned on the blade itself, after application of a $100 \mathrm{~kg}$ load on the free end. The strain was acquired by the BOTDA sensor at a spatial resolution of $1 \mathrm{~m}$ and an acquisition rate of $\sim 15 \mathrm{~Hz}$. We report in Figure 5(a) the dynamic strain measured at the clamped end (blue curve) and free end (red curve) of the blade and in Figure 5(b) the corresponding Fourier spectra. As expected, the amplitude of the strain is large on the clamped end of the blade, while being minimum at the free end. The first natural frequency $(f \sim$ $0.9 \mathrm{~Hz}$ ) of the instrumented blade is clearly observed. The second resonant peak, visible at $\sim 1.8 \mathrm{~Hz}$, has to be attributed to the nonlinear response of our sensor for high (several hundreds of $\mu \varepsilon$ ) dynamic strain levels [8].

As a second step, the dynamic strain was acquired after changing the load condition, in order to verify the capability of our sensor to detect the changes in natural frequencies resulting from structural modifications. A $150 \mathrm{~kg}$ load was considered in this new test. The results are shown in Figure 6.

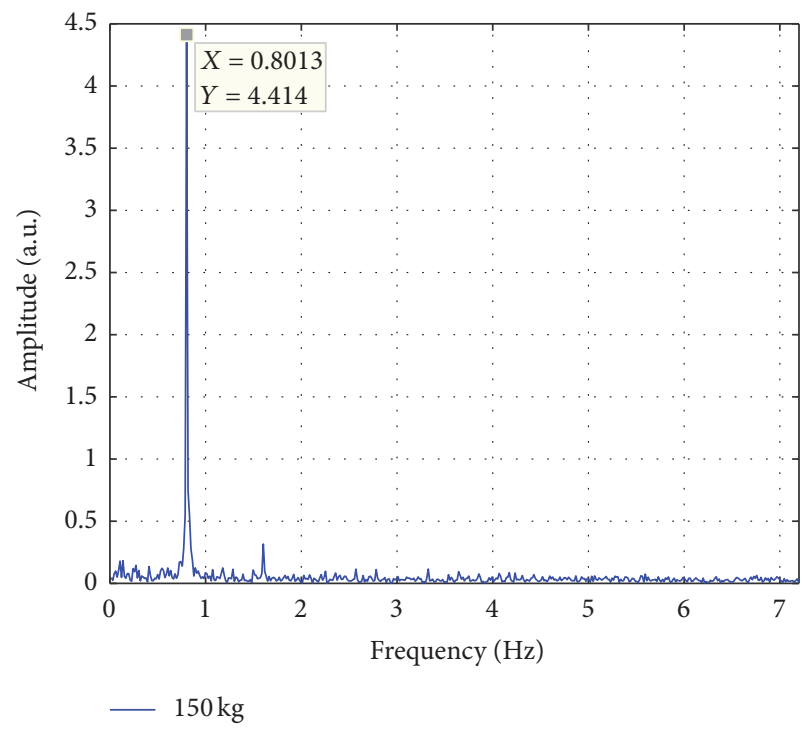

FIGURE 6: Fourier spectrum of the sensor signal at a fiber position close to the clamp (load weight $=150 \mathrm{~kg})$.

A redshift of the resonance frequency $(\sim 0.8 \mathrm{~Hz})$ is measured, in agreement with the higher applied load. We also detect a second peak at doubled frequency $(\sim 1.6 \mathrm{~Hz})$, which is still to be attributed to the nonlinear behavior of our sensor at high strain levels. These tests demonstrate the ability of our sensor to perform vibration tests on flexible structures, supporting the design optimization and control process of the structures.

\section{Conclusions}

In conclusion, static and dynamic strain was acquired at various positions along a $14 \mathrm{~m}$ WT blade with a spatial 
resolution of $1 \mathrm{~m}$. As the signal acquisition and data subsequent analysis is completed in about one minute, the system provides a practically thorough spatial-temporal monitoring. The presented setup can be used to support the design optimization and control process of the structures. Future developments of this technology relate to the possibility of embedding the optical fiber in composite materials in order to realize a smart structure.

\section{Conflicts of Interest}

The authors declare that they have no conflicts of interest.

\section{References}

[1] X. Bao and L. Chen, "Recent progress in distributed fiber optic sensors," Sensors, vol. 12, no. 7, pp. 8601-8639, 2012.

[2] L. Thévenaz, "Brillouin distributed time-domain sensing in optical fibers: state of the art and perspectives," Frontiers of Optoelectronics in China, vol. 3, no. 1, pp. 13-21, 2010.

[3] F. Zhang, Y. Li, Z. Yang, and L. Zhang, "Investigation of wind turbine blade monitoring based on optical fiber Brillouin sensor," in Proceedings of the 1st International Conference on Sustainable Power Generation and Supply (SUPERGEN '09), IEEE, Nanjing, China, April 2009.

[4] V. Sotoudeh, "Fiber-Optic Defect and Damage Locator System for Wind Turbine Blades," Intelligent Fiber Optic Systems Corporation (IFOS), 2010.

[5] S. M. Klute, A. K. Sang, D. K. Gifford, and M. E. Froggatt, "Defect detection during manufacture of composite wind turbine blade with embedded fiber optic distributed strain sensor," in Proceedings of the SAMPE Tech Conference and Exhibition: Developing Scalable Materials and Processes for Our Future, October 2011.

[6] J. Xu, Y. Dong, and H. Li, "Research on fatigue damage detection for wind turbine blade based on high-spatial-resolution DPPBOTDA," in Sensors and Smart Structures Technologies for Civil, Mechanical, and Aerospace Systems 2014, 906130, vol. 9061 of Proceedings of SPIE, San Diego, Calif, USA, March 2014.

[7] G. C. Larsen, M. H. Hansen, A. Baumgart, and I. Carlén, "Modal analysis of wind turbine blades" Risoe-R 1181(EN), Forskningscenter Risoe, Roskilde, Denmark, 2002.

[8] R. Bernini, A. Minardo, and L. Zeni, "Dynamic strain measurement in optical fibers by stimulated brillouin scattering," Optics Letters, vol. 34, no. 17, pp. 2613-2615, 2009.

[9] A. Minardo, A. Coscetta, S. Pirozzi, R. Bernini, and L. Zeni, "Modal analysis of a cantilever beam by use of Brillouin based distributed dynamic strain measurements," Smart Materials and Structures, vol. 21, no. 12, Article ID 125022, 2012.

[10] A. Minardo, A. Coscetta, S. Pirozzi, R. Bernini, and L. Zeni, "Experimental modal analysis of an aluminum rectangular plate by use of the slope-assisted BOTDA method," Smart Materials and Structures, vol. 22, no. 12, Article ID 125035, 2013. 


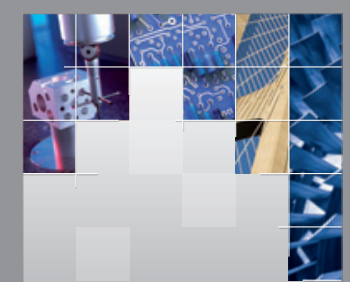

\section{Enfincering}
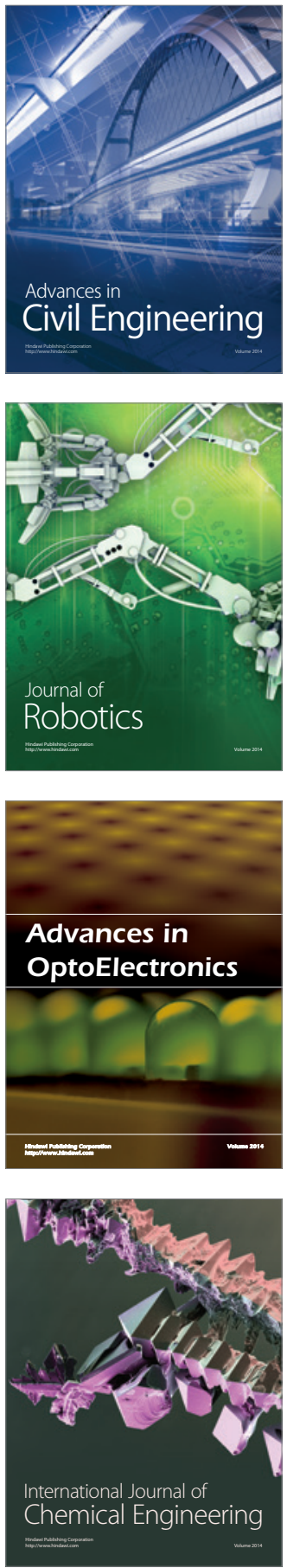



The Scientific World Journal



\section{Hindawi}

Submit your manuscripts at

https://www.hindawi.com
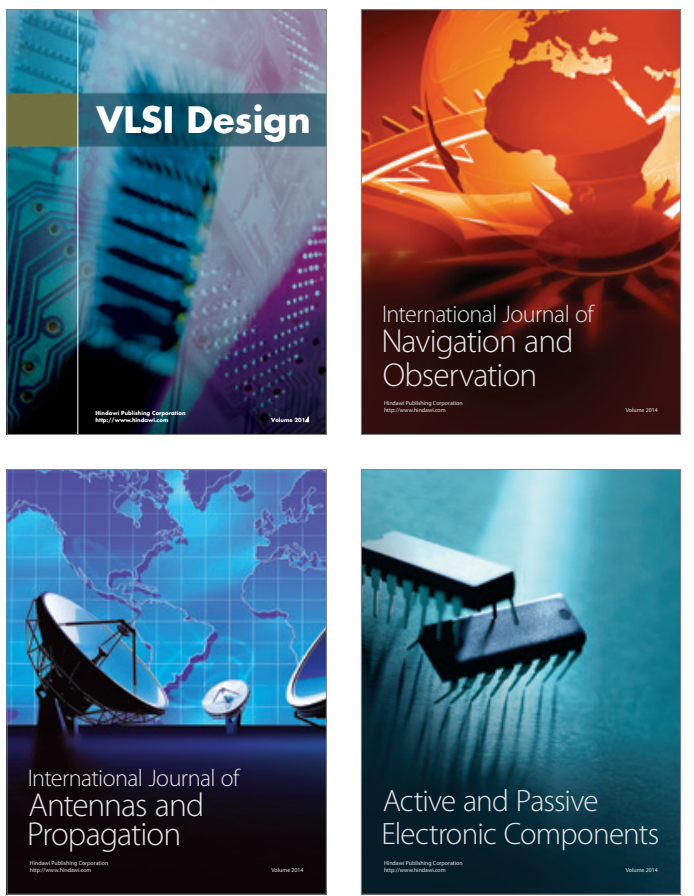
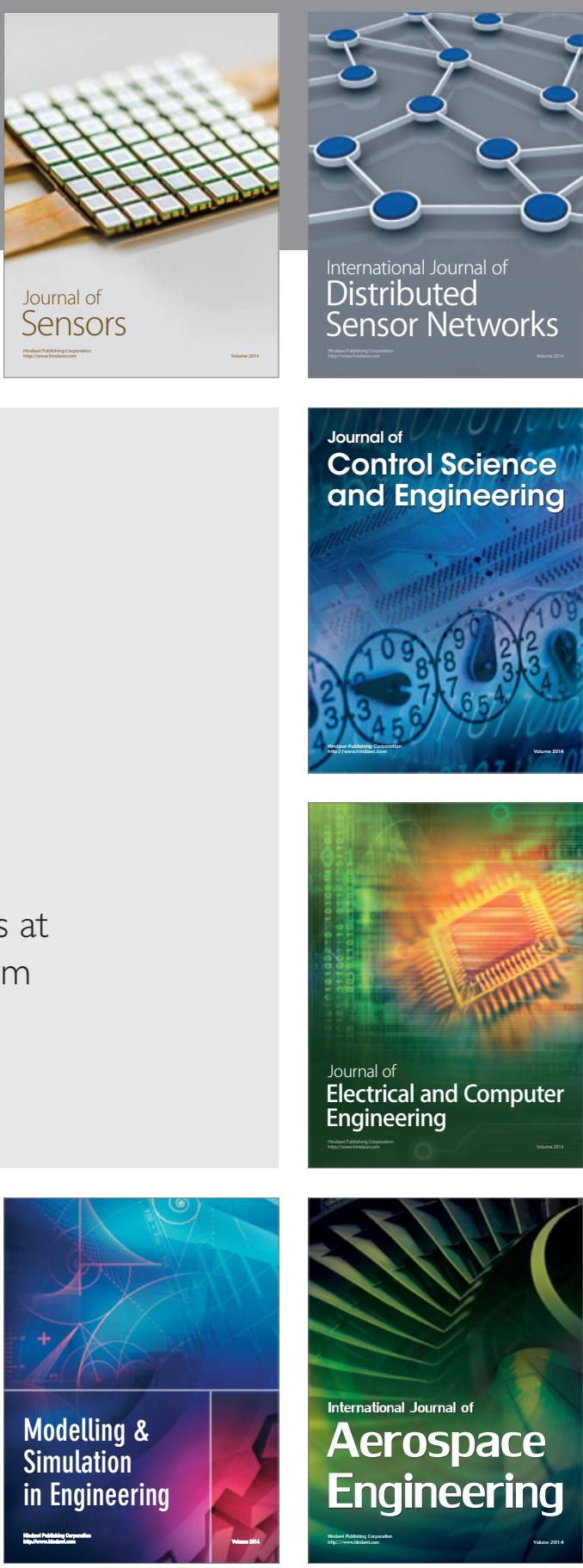

International Journal of

Distributed

Sensor Networks

$-$

Joumal of

Control Science

and Engineering
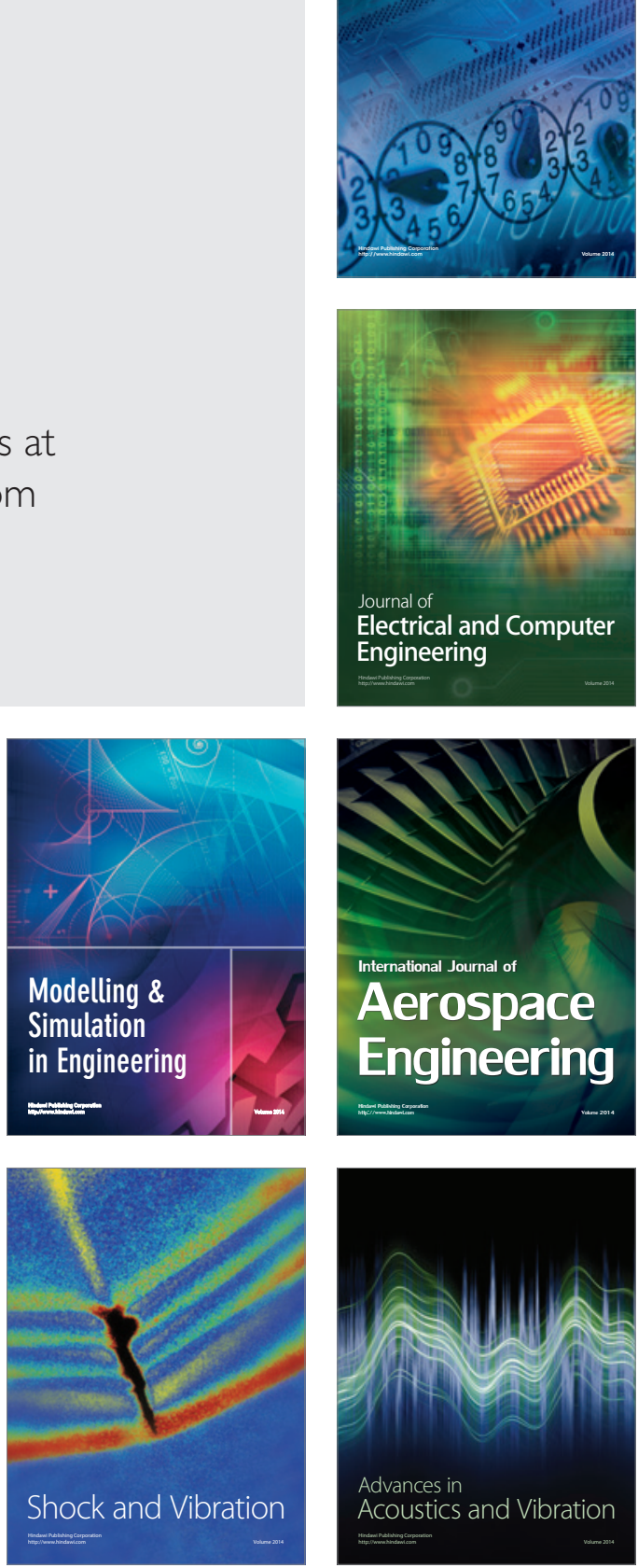